\title{
Origin of Dynamic Heterogeneities in Miscible Polymer Blends: A Quasielastic Neutron Scattering Study
}

\author{
S. Hoffmann, ${ }^{1,2}$ L. Willner, ${ }^{1}$ D. Richter, ${ }^{1}$ A. Arbe,${ }^{3}$ J. Colmenero, ${ }^{3}$ and B. Farago ${ }^{2}$ \\ ${ }^{1}$ Institut für Festkörperforschung, Forschungszentrum Jülich, 52425 Jülich, Germany \\ ${ }^{2}$ Institut Laue-Langevin, 156X, 38042 Grenoble CEDEX, France \\ ${ }^{3}$ Departamento de Física de Materiales y Centro Mixto CSIC-UPV/EHU, Universidad del País Vasco, \\ Apartado 1072, 20080 San Sebastián, Spain
}

(Received 15 July 1999)

\begin{abstract}
In order to investigate the origin of the often invoked nanoheterogeneities in miscible polymer blends, we have performed quasielastic neutron scattering experiments on the component dynamics within the miscible polymer blend polyisoprene/polyvinyl ether including the pure components as a reference. We find that the apparent local heterogeneities observed by spectroscopic techniques originate from the chain specific crossover properties between entropy driven and local chain dynamics and are, thus, a purely dynamical phenomenon.
\end{abstract}

PACS numbers: $61.41 .+\mathrm{e}, 61.12 . \mathrm{Ex}, 61.25 . \mathrm{Hq}$

The study of thermodynamic and structural properties of polymer blends is a very active field in polymer physics. Recently also dynamical aspects like the socalled "dynamic miscibility," i.e., the question how the chain dynamics is altered upon blending, came into focus [1-10]. Mainly on the basis of results from spectroscopic techniques many workers have invoked the existence of nanoheterogeneities in thermodynamically miscible blends and disordered block copolymer systems [2-6]. Among the different materials studied, the miscible blend of polyisoprene and polyvinyl ether (PI/PVE) has been investigated particularly well and can be considered as a model system [3-7,9]. 2D NMR revealed [3] that, though the system is homogeneous (Flory Huggins parameter $\chi<0$ ), the two polymers exhibit significantly different mobilities. By dielectric spectroscopy several groups have found two separate relaxations in the miscible regime (e.g., $[5,6])$. This finding is supported by recent quasielastic neutron scattering (QENS) studies, where at large momentum transfers distinctly different QENS patterns are observed for the two components [4,9]. Such findings are not restricted to PI/PVE system but have been observed also in several other miscible polymer blends (e.g., $[2,8]$ ). Finally, studies by forward recoil spectroscopy [10] on polystyrene/poly(xylenyl ether) blends resulted in grossly different component diffusion coefficients, indicating very different friction coefficients for the two components in this particular blend.

Recently two theoretical pictures were brought forward: Khokhlov et al. [11] proposed a nonlocal $\chi$ parameter due to local entropy differences which should lead to microphase separation in blends as in diblock copolymer systems. Based on the concept of cooperativity volumes, Kumar et al. [5] developed a theory of concentration fluctuations predicting bimodal distribution functions of local environments.

Nanoheterogeneities invoke a mesoscopic length scale beyond which the system becomes homogeneous dynami- cally. In order to investigate the mechanisms behind the suggested crossover from locally heterogeneous to mesoscopically homogeneous dynamics in blends, QENS is particularly well suited, because (i) it provides direct information on the spatial evolution of motional processes on the proper time and length scales and (ii) without disturbing the system by proper hydrogen/deuterium $(\mathrm{h} / \mathrm{d})$ labeling, its sensitivity can be made chain specific.

In this Letter we present QENS results on a PI/PVE blend over nearly 2 orders of magnitude in momentum transfer $Q[Q=4 \pi \sin (\theta / 2) / \lambda, \lambda$ : neutron wavelength, $\theta$ : scattering angle]. We observe distinctly different chain motions on local scales changing to nearly homogeneous dynamics on the scale of the entropy driven Rouse modes with a crossover at around $Q \approx 0.2 \AA^{-1}$ corresponding to length scales of about $30 \AA$.

On intermediate length scales the dynamics of a polymer chain in the melt to a good approximation can be described in terms of the Rouse model $[12,13]$. This model treats the dynamics of a Gaussian chain in a heat bath. Thereby it implicitly assumes that on the considered length and time scales all forces from local potentials which are related to the individual chemical nature of a given polymer have already decayed. Then only entropic forces originating from the conformational chain entropy drive the dynamics. At scales $Q R_{E} \gg 1$, where $R_{E}$ is the chain end-to-end distance, the normalized single chain dynamic structure factor $S$ for this model can be written as a function of a scaling variable $u=Q^{2} \sqrt{W \ell^{4} t}$ combining spatial and temporal scales [12],

$$
\begin{aligned}
S(u)=\int_{0}^{\infty} d s \exp \{ & -s-\frac{u}{3 \pi} \int_{0}^{\infty} \frac{\cos \left(\frac{6 x s}{u}\right)}{x^{2}} \\
& \left.\times\left[1-\exp \left(-x^{2}\right)\right] d x\right\} .
\end{aligned}
$$

Thereby $W=3 k_{B} T /\left(\zeta \ell^{2}\right)$ is the elementary Rouse frequency. It is given by the ratio of the entropic force 
$3 k_{B} T / \ell^{2}$ and the friction coefficient $\zeta . \ell^{2}$ is the mean squared segment length.

We remark that at scales beyond the Rouse regime entanglements lead to a retardation of the decay of $S(u)$ compared to Eq. (1) [12]. In order to ensure that only results in the applicability range of Eq. (1) are considered, the experimental time range has to be restricted to times smaller than the entanglement time $\tau_{e}=d^{4} /\left(\pi^{2} W \ell^{4}\right)$ (typically $\left.t<\tau_{e} / 4\right) . d$ is the entanglement distance (PI: $d=52 \AA$; PVE: $d=50 \AA$ ) [13].

At shorter scales the spatial region of the $\alpha$ process is entered. For a theoretical description of the chain dynamics no general theory exists. In analogy to the spectroscopic results we model the self-correlation function $S_{\text {self }}(Q, \omega)$ ( $\omega$ : energy transfer) by the Fourier transformed of a Kohlrausch-Williams-Watts (KWW) expression [14]

$$
S_{\text {self }}(Q, \omega)=F T\left\{\exp \left[-\left(\frac{t}{\tau_{\mathrm{KWW}}(Q)}\right)^{\beta}\right]\right\} .
$$

It has been found that for polymers in general $\tau_{\mathrm{Kww}}(Q) \sim$ $Q^{-n}(n \approx 2 / \beta)$ [14]. Furthermore the stretching parameter $\beta$ was observed to be closely related to the corresponding values found by dielectric spectroscopy (PI: $\beta=0.4$; PVE: $\beta=0.43$ ). After earlier macroscopic studies [15] the transition zone between both regimes has been recently investigated in detail by neutron spin echo (NSE) on polyisobutylene [16] revealing rich and complex crossover phenomena.

For the experiments hydrogenated and fully deuterated PI and PVE materials were synthesized anionically. In order to study the single chain structure factor, samples with a $10 \%$ labeled (protonated) chain fraction in otherwise deuterated matrices were prepared [hPI/dPI; hPVE/dPVE; $\mathrm{hPI} /(\mathrm{dPI} / \mathrm{dPVE})$ and $\mathrm{hPVE} /(\mathrm{dPI} / \mathrm{dPVE})]$. The number averaged molecular weights were $M_{n}^{\mathrm{hPI}}=82000 ; M_{n}^{\mathrm{dPI}}=$ $91000 ; \quad M_{n}^{\mathrm{hPVE}}=81000$, and $M_{n}^{\mathrm{dPVE}}=90000$ with $M_{W} / M_{n}<1.02 \quad\left(M_{W}\right.$ : weight averaged molecular weight). The component wise study of the self-correlation function dwells on the dominant incoherent scattering from a protonated polymer in a blend at higher $Q$. For these studies the following samples were prepared: hPI, hPVE, $\mathrm{hPI} / \mathrm{dPVE}$, and hPVE/dPI. In the blends the total polymer composition amounted always to 50/50 volume percent.

To cover a maximum space-time range, a combination of different spectrometers [NSE spectrometers at low $Q$, backscattering (BS) spectrometer at high $Q$ ] and the consistent application of the time temperature superposition principle was necessary. The investigations of the single chain dynamic structure factors were performed at the NSE spectrometer IN11 at the Institute Laue-Langevin (ILL) in Grenoble. Seven different $Q$ values in the range $0.05 \leq$ $Q \leq 0.2 \AA^{-1}$ at three different temperatures $(T=418$, 368 , and $330 \mathrm{~K}$ ) were studied. Figure 1 presents spectra at $418 \mathrm{~K}$ from the two pure polymers as well as from PVE and PI in the blend as a function of the Rouse scaling variable $Q^{2} \sqrt{t}$. In all cases the data follow very well the Rouse scaling and, at least for smaller values of the scaling

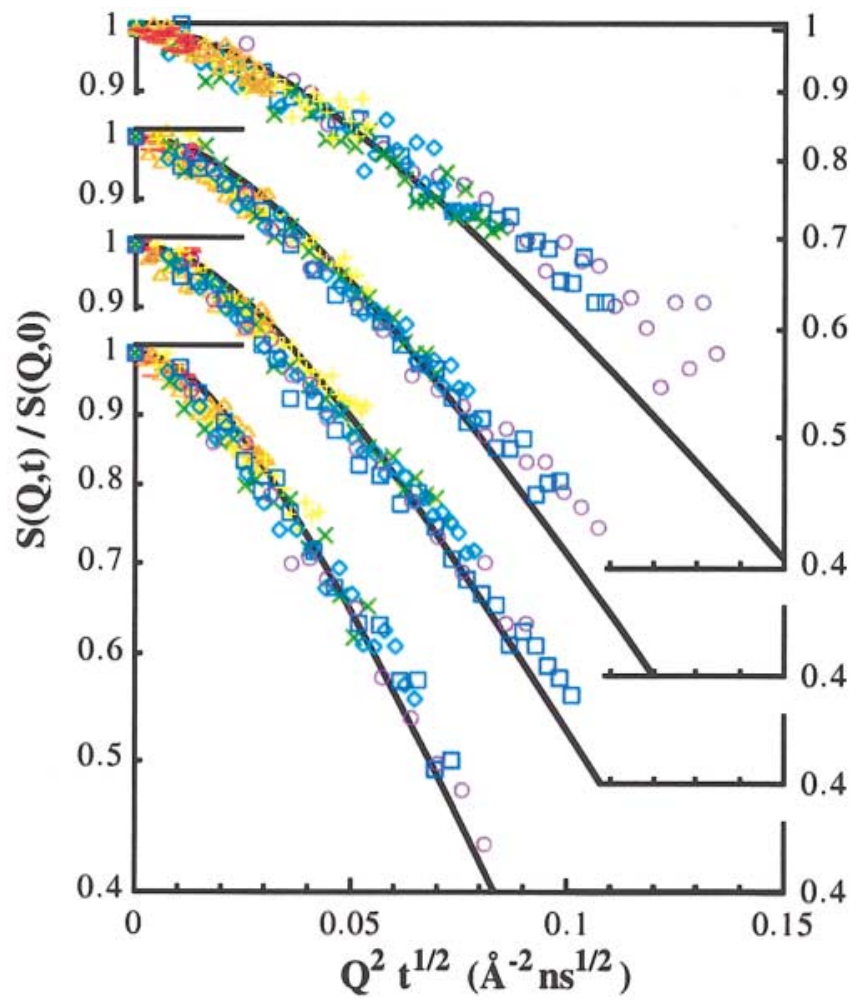

FIG. 1 (color). NSE results at $418 \mathrm{~K}$ on the single chain dynamic structure factors from PVE, PVE in PI/PVE, PI in PI/PVE, and PI (from above) (-: $0.05 \AA^{-1} ; \triangle: 0.077 \AA^{-1}$; +: $0.01 \AA^{-1} ; \times: 0.13 \AA^{-1} ; \diamond: 0.15 \AA^{-1} ; \square: 0.18 \AA^{-1}$; $\left.\bigcirc: 0.20 \AA^{-1}\right)$. The data are scaled according to Eq. (1). Solid lines: Rouse structure factors [Eq. (1)].

variable, also the Rouse dynamic structure factor [Eq. (1)] provides a satisfying description of the experimental results. Comparing the decay of the relaxation functions, we realize that (i) the time scale of the Rouse motion in pure PI and PVE is significantly different, and that (ii) the relaxation curves for PI and PVE in the blend are practically identical, indicating close to homogeneous dynamics. For the Rouse rates $\left(W \ell^{4}\right)$ the fits yield PI: $12600 \AA^{4} \mathrm{~ns}^{-1}$; PVE: $3800 \AA^{4} \mathrm{~ns}^{-1}$; PI in blend: $7500 \AA^{4} \mathrm{~ns}^{-1}$; and PVE in blend: $6100 \AA^{4} \mathrm{~ns}^{-1}$.

$Q$-dependent Rouse rates are obtained by fitting each spectrum separately. For a comparison with the BS data (see below) the obtained values for $W \ell^{4}(Q)$ may be converted into average relaxation times for the Rouse selfcorrelation function by $\tau_{\mathrm{av}}^{R}(Q)=18 \pi Q^{-4} /\left[W \ell^{4}(Q)\right]$ [12].

In order to relate to the $\mathrm{BS}$ results at $330 \mathrm{~K}$, temperature shift factors $a_{T}$ covering the appropriate $T$ range were measured using the very high resolution NSE spectrometer IN15 at the ILL. Experiments were performed at $Q=$ $0.15 \AA^{-1}$ on all four samples for nine different temperatures covering a temperature range $305 \leq T \leq 422 \mathrm{~K}$. Employing the time temperature superposition principle, Fig. 2 presents the obtained spectra as a function of the rescaled time $t / a_{T}$ (reference temperature $368 \mathrm{~K}$ ). The respective shift factors for the polymers in the blend agree 


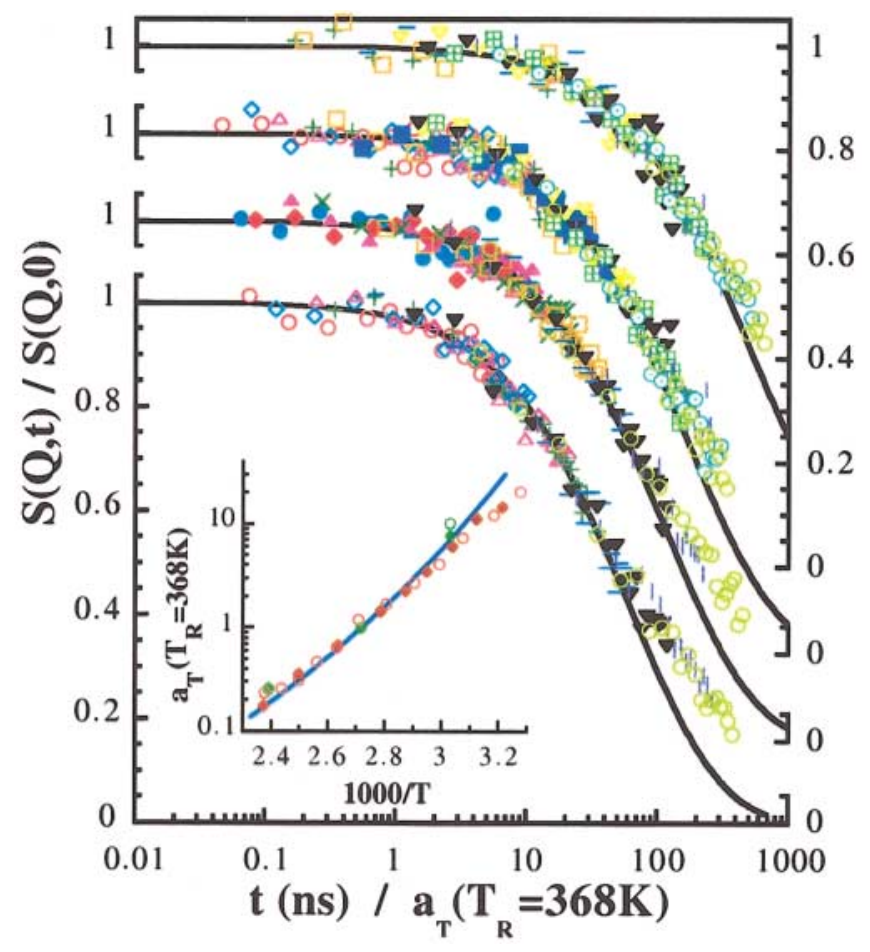

FIG. 2 (color). Single chain dynamic structure factors at $Q=$ $0.15 \AA^{-1}$ vs the rescaled time $t / a_{T}$. From above PVE, PVE in PI/PVE, PI in PI/PVE, and PI. Different symbols refer to different temperatures in the interval $305 \leq T \leq 422 \mathrm{~K}$. Solid lines: Rouse structure factors [Eq. (1)]. Inset: Temperature shift factors $a_{T}$ for the polymers in the blend ( $\diamond$ : PI in PI/PVE; $\mathrm{O}$ : PVE in PI/PVE; solid line: dielectric results).

very well with the corresponding dielectric results for the end-to-end vector relaxation (normal mode) for PI [17] (see inset in Fig. 2). Furthermore we emphasize that the shift factors for PVE and PI in the blend follow an identical temperature dependence. Thus, the finding that at $418 \mathrm{~K}$ on the level of the Rouse modes the blend dynamics is homogeneous is not fortuitous but holds over the entire temperature range investigated.

The relaxation spectra for the four polymer systems at higher $Q$ 's $\left(0.2 \leq Q \leq 1.9 \AA^{-1}\right)$ were investigated at the BS spectrometer at the FRJ-2 reactor in Jülich. The temperature range was $270 \leq T \leq 340 \mathrm{~K}$. Here we concentrate on the $330 \mathrm{~K}$ data, where BS reveals results covering a maximum $Q$ range. Figure 3 displays spectra for the four polymer systems at a representative $Q$ value $\left(Q=1.1 \AA^{-1}\right)$. The data are compared to the instrumental resolution function determined with the samples at $10 \mathrm{~K}$. Starting from PI, where the spectrum is broadest, the spectra narrow successively in going to $\mathrm{hPI} / \mathrm{dPVE}$, then to $\mathrm{hPVE} / \mathrm{dPI}$, and finally to pure PVE. In distinct contrast to the Rouse regime the spectra from the two components in the blend show a strongly different broadening implying significantly different or heterogeneous relaxation rates.

The data were fitted to Eq. (2) keeping the stretching parameter $\beta$ to its dielectric value. The solid lines in Fig. 3 display the resulting curves. These fits lead to the $Q$-dependent characteristic relaxation times $\tau_{\mathrm{KWw}}(Q)$

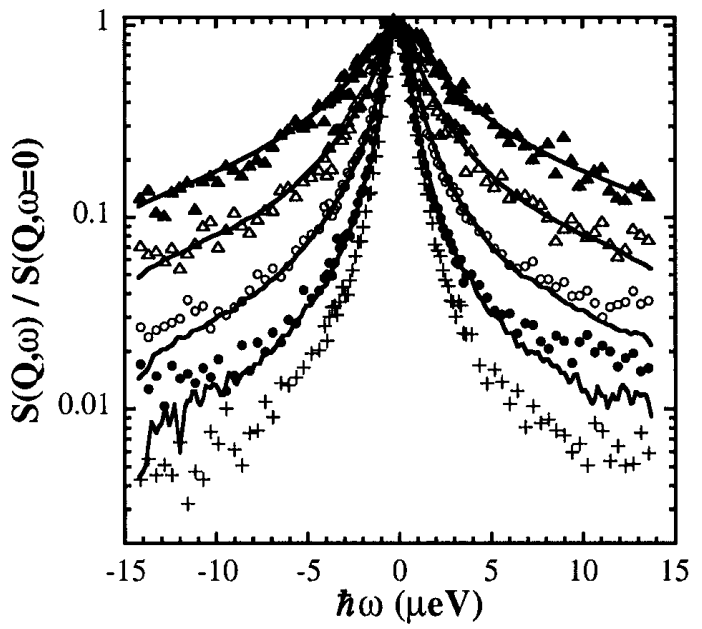

FIG. 3. BS results at $330 \mathrm{~K}$ and at $Q=1.1 \AA^{-1}$. From above PI, PI in PI/PVE, PVE in PI/PVE, PVE, and instrumental resolution. Solid lines: fit with Eq. (2).

which are converted to average relaxation times by $\tau_{\mathrm{av}}=$ $\tau_{\mathrm{Kww}} \Gamma(1 / \beta) / \beta$ (see Fig. 4). The error bars include systematic errors due to multiple scattering contributions particularly important at low $Q$ [14].

Figure 4(a) presents the $Q$-dependent average relaxation times for the four samples at $330 \mathrm{~K}$. For this presentation the average Rouse relaxation times $\tau_{\mathrm{av}}^{R}(Q)$ obtained at $418 \mathrm{~K}$ were shifted to $330 \mathrm{~K}$ applying the shift factors of Fig. 2. Figure 4(b) emphasizes the $Q$-dependent crossovers to local dynamics in plotting $\tau_{\text {av }} Q^{4}$. Thereby the intrinsic $Q$ dependence of the Rouse relaxation is taken out. We first concentrate on the pure homopolymer melts. At low $Q$ (PI: $Q \leq 0.2 \AA^{-1}$; PVE: $Q \leq 0.15 \AA^{-1}$ ) the dynamics of both polymers is in accordance with the Rouse model [solid lines in Figs. 4(a) and 4(b)]. Moving to shorter scales (higher $Q$ ) for PI $\tau_{\text {av }}(Q)$ decreases only slightly less than an extrapolation of the Rouse relaxation would prescribe. In Fig. 4(b) this reveals itself as a slight upturn of $\tau_{\text {av }} Q^{4}$ vs $Q$. In contrast to PI, approaching local scales the time scale of motion in PVE is significantly retarded compared to the Rouse-like $Q^{-4}$ behavior. In Fig. 4(b) this crossover shows itself in terms of a strong increase of $\tau_{\mathrm{av}} Q^{4}$ towards high $Q$ (upper dashed line). As a consequence, while in the Rouse regime at $330 \mathrm{~K}$ the time scale of motion between PI and PVE differs by a factor of 15 , at short scales this difference increases to about 150 . Thus, the shape of the dynamic crossover is chain specific and relates to individual chain properties such as the rotational potential and steric hindrances.

We now turn to the blend. As already pointed out above, on the spatial scale of the Rouse motion the chain dynamics in the blend is practically homogeneous [low $Q$ part in Figs. 4(a) and 4(b)] in the whole investigated temperature range-both respective shift factors are identical (see Fig. 2). On the other hand, towards higher $Q$ or shorter scales, both components in the blend display 


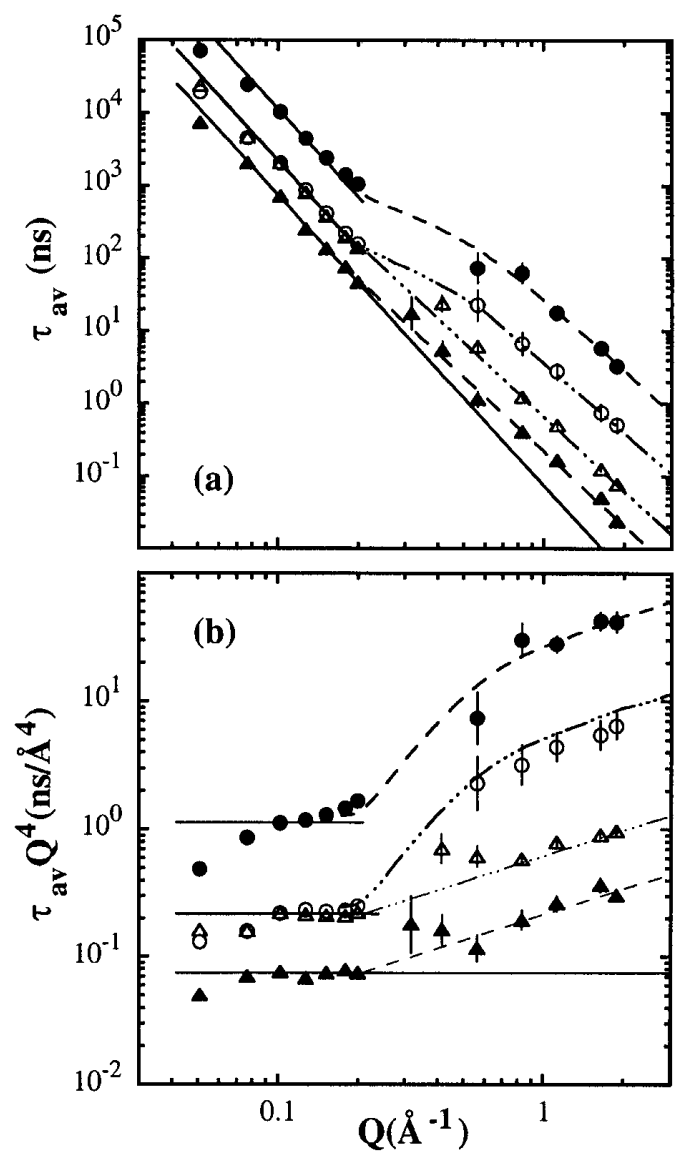

FIG. 4. (a) Characteristic times $\tau_{\mathrm{av}}$ at $330 \mathrm{~K}$ (PI: $\boldsymbol{\Delta}$; PI in PI/PVE: $\triangle$; PVE in PI/PVE: ; PVE: $)$ ). (b) $\tau_{\text {av }} Q^{4}$ vs $Q$. Solid lines: Rouse prediction valid for low $Q$; dashed and dashdotted lines are guides to the eye.

the same crossover to local dynamics as in the respective homopolymer melts. This may be seen directly from Fig. 4(b). There, the dash-dotted lines describe the $Q$ dependence of $\tau_{\mathrm{av}} Q^{4}$ for the two components in the blend. They are the result of shifting the respective curves for the two homopolymers such that they agree in the homogeneous Rouse regime. Obviously, such shifted lines describe the component crossovers in the blend very well. Thus, apart from a general shift in time scale, both components retain their individual crossover behaviors also in the blend.

This observation explains the apparent dynamic heterogeneities found by local probes: They mainly originate from the respective different crossover properties of the two components from Rouse to local dynamics. Though the Rouse dynamics are homogeneous, the different crossovers at $330 \mathrm{~K}$ cause a 1 order of magnitude difference in the local characteristic times. This value decreases towards higher temperatures, and, following dielectric and NMR investigations, will increase towards lower temperatures. With this finding we, thus, may classify the dynamic heterogeneities as a purely dynamical phenomenon related to the respective homopolymer dynamics.

Finally, the dynamic crossover between both regimes, the Rouse regime and the regime of local relaxation, takes place at $Q$ values around $Q \approx 0.2 \AA^{-1}$ implying a spatial crossover length of $2 \pi / Q \approx 3 \mathrm{~nm}$, a value very close to NMR results [2]. However, the mechanism behind the phenomenon is quite different from that supposed in the interpretation of the NMR results, where nanoheterogeneities were invoked. The neutron results demonstrate that we deal with a dynamic crossover from universal entropy driven dynamics common for both components to heterogeneous local motion specific for the two homopolymers.

A. A. and J.C. acknowledge support from the following projects: DGICYT, PB97-0638; GV, EX 1998-23; UPV/ EHU, 206.215-G20/98. Support from "Donostia International Physics Center" is also acknowledged by A. A., J. C., and D. R.

[1] G. Katana, E. W. Fischer, Th. Hack, V. Abetz, and F. Kremer, Macromolecules 28, 2714 (1995).

[2] K. Schmidt-Rohr, J. Clauss, and H. W. Spiess, Macromolecules 25, 3273 (1992).

[3] G.-C. Chung, J. A. Kornfield, and S.D. Smith, Macromolecules 27, 964 (1994); 27, 5729 (1994).

[4] G. Fytas, G. Meier, and D. Richter, J. Chem. Phys. 105, 1208 (1996).

[5] S. K. Kumar, R. H. Colby, S. H. Anastiasadis, and G. Fytas, J. Chem. Phys. 105, 3777 (1996).

[6] F. Alvarez, A. Alegría, and J. Colmenero, Macromolecules 30, 597 (1997).

[7] S. Adams and D. B. Adolf, Macromolecules 32, 3136 (1999).

[8] I. Cendoya, A. Alegría, J. M. Alberdi, J. Colmenero, H. Grimm, D. Richter, and B. Frick, Macromolecules 32, 4065 (1999).

[9] A. Arbe, A. Alegría, J. Colmenero, S. Hoffmann, L. Willner, and D. Richter, Macromolecules 32, 7572 (1999).

[10] R. J. Composto, E. J. Kramer, and D. M. White, Polymer 31, 2320 (1990).

[11] A. R. Khokhlov and J. Ya Erukhimovich, Macromolecules 26, 7195 (1993).

[12] M. Doi and S. F. Edwards, The Theory of Polymer Dynamics (Clarendon, Oxford, 1986).

[13] B. Ewen and D. Richter, Advances in Polymer Science (Springer-Verlag, Berlin/Heidelberg, 1997), Vol. 134.

[14] A. Arbe, J. Colmenero, M. Monkenbusch, and D. Richter, Phys. Rev. Lett. 81, 590 (1998).

[15] T. Inoue and K. Osaki, Macromolecules 29, 1595 (1996).

[16] D. Richter, M. Monkenbusch, J. Allgeier, A. Arbe, J. Colmenero, B. Farago, Y. Cheol Bae, and R. Faust, J. Chem. Phys. 111, 6107 (1999).

[17] S. Hoffmann, L. Willner, D. Richter, A. Alegría, A. Arbe, and J. Colmenero (to be published). 